\title{
Bilateral recurrent orbital inflammation associated with retroperitoneal fibrosclerosis
}

\author{
ADOLPH A SCHONDER, ROBERT C CLIFT, JOHN W BROPHY, \\ AND LEAR W DANE
}

From 350 W Sixth Street, Suite A-1, Reno, NV 89503, USA

SUMMARY Orbital inflammation initially presented in a patient who five years later developed renal obstruction from retroperitoneal fibrosclerosis. Biopsy revealed infiltration of chronic lymphocytic cells in the orbit and dense fibrous connective tissue in the pelvis. Episodes of proptosis were controlled with systemic steroids and non-steroidal anti-inflammatory agents. The hydronephrosis was relieved by intraperitonisation of the ureters. Similarities to previously reported cases are discussed. This case shows the importance of searching for systemic disease in patients with orbital inflammatory disease and informing the patients of the possibility of future systemic manifestations of their disease.

Unilateral or bilateral orbital inflammation associated with varying degrees of proptosis, fibrosis, and limitation of ocular motility (orbital pseudotumour) has been observed to occur in individuals who also have systemic diseases such as sarcoidosis, Wegener's granulomatosis, histicytosis- $\mathrm{X}$, thyroid disease, Waldenström's macroglobulinaemia and polyarteritis nodosa. ${ }^{12}$ Comings and co-workers ${ }^{3}$ were the first to report the association of orbital inflammation with retroperitoneal fibrosis, and, because they observed the condition in two brothers, they suggested the name 'familial multifocal fibrosclerosis.' We wish to report a non-familial case in which the initial episode of orbital inflammatory disease preceded by five years the development of obstructive ureteral-renal disease secondary to retroperitoneal fibrosis. This case illustrates the importance of searching for evidence of systemic disease in patients with orbital inflammatory disorders and of informing the patients of the potential for future systemic manifestations of their disease.

\section{Case report}

A 36-year-old white man presented on 26 January 1975 with sudden onset of proptosis, chemosis, and ophthalmoplegia of his left eye. These symptoms were preceded by a two-week history of a mild febrile illness and weakness. The patient had previously

Correspondence to Adolph A Schonder MD. been in good health and gave no history of ingestion of medications such as methysergide. He had no history of chronic gastroenteritis. His three sisters and one brother were in good health. His mother had surgery at the age of 30 to remove fibrous tissue from her chest that obstructed her swallowing. No further symptoms developed, and she is well at age 68 . At the time of his initial ocular examination the patient's uncorrected visual acuity was $20 / 25$ in each eye. The pupils were equal and reactive. The conjunctiva of the left eye was hyperaemic and chemotic. The left globe and orbit were tender to palpation, and obvious left proptosis was present. The left globe was depressed. There was initially limitation of upgaze only, but this progressed to total ophthalmoplegia within 24 hours.

On admission to hospital his laboratory tests were found to be normal, except for a raised Westergren sedimentation rate of $41 \mathrm{~mm} / \mathrm{h}$. Bilateral carotid arteriograms were normal. Orbital tomograms showed slight bony erosion of the left greater wing of the sphenoid.

On 4 February 1975 a Krönlein decompression and exploration of the left orbit was done. No localised mass was found. Three pieces of diffusely inflamed soft tissue and lacrimal gland were obtained. Histological examination (Fig. 1) revealed focal areas of chronic lymphocytic infiltration compatible with a diagnosis of lymphoid pseudotumour of the orbit. The tissue did not appear to be fibrotic.

$80 \mathrm{mg}$ of oral prednisone was started daily, and the 
Fig. 1 Biopsy of benign lacrimal gland with focal benign lymphocytic infiltrates. ( $H$ and $E$, $\times 300$ ).

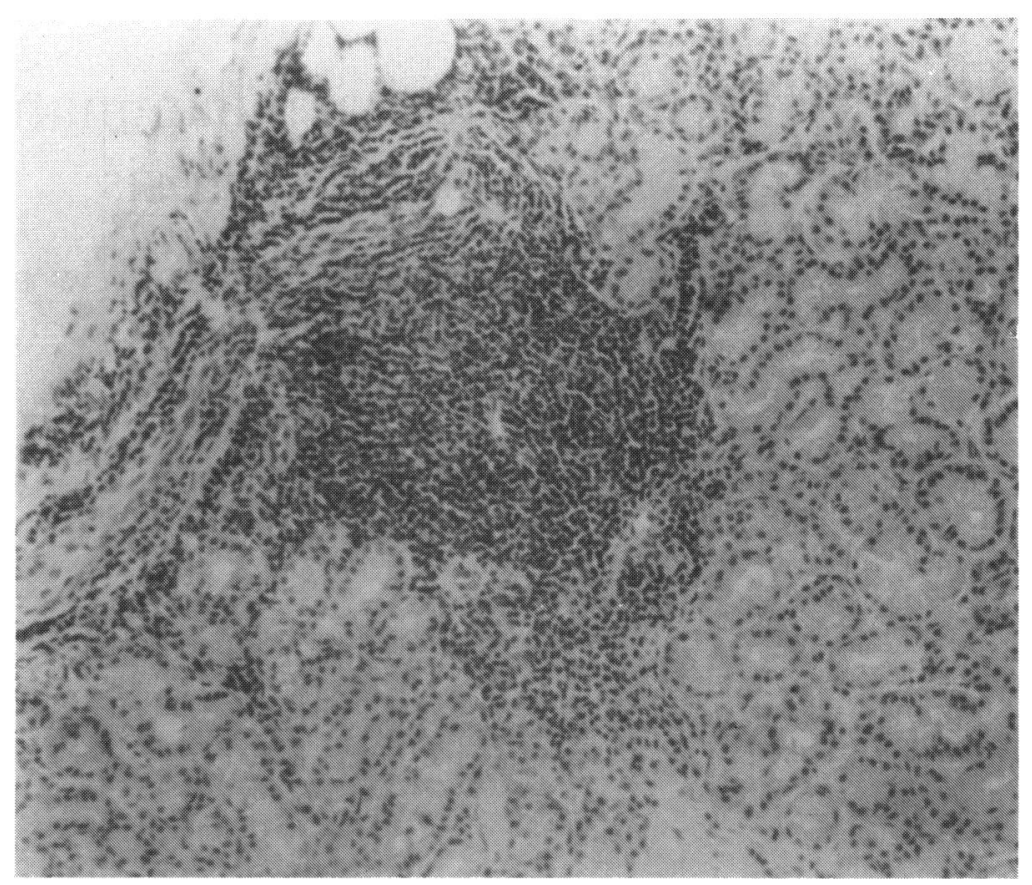

patient was discharged from hospital on 7 February 1975 . The oral prednisone was gruadually tapered to $25 \mathrm{mg}$ every other day. On 28 February 1975 the patient developed pain and swelling about his right eye. No ophthalmoplegia was present. Repeat orbital tomograms were normal. Prednisone was increased to $80 \mathrm{mg}$ daily, and as the right periocular pain and swelling subsided the prednisone was slowly tapered until it was discontinued in July 1975 . He was seen intermittently for routine ocular examinations
Fig. 2 Biopsy of perirectal mass with benign fibroconnective tissue.

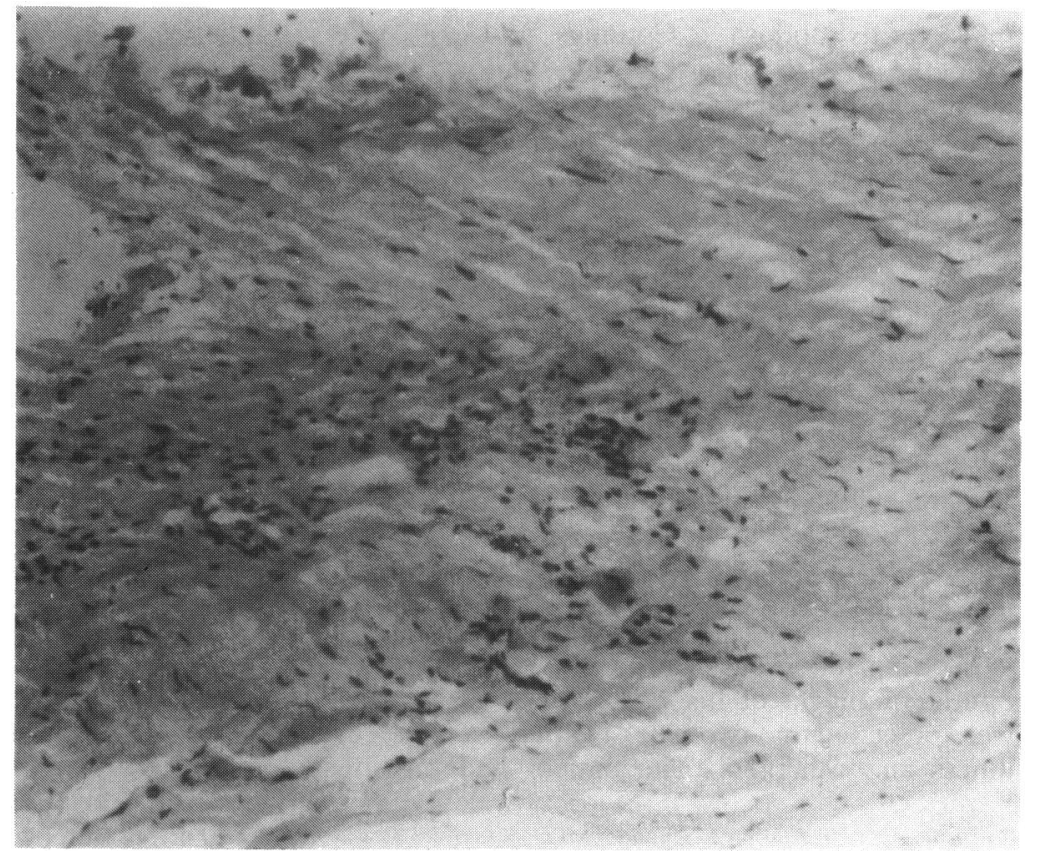




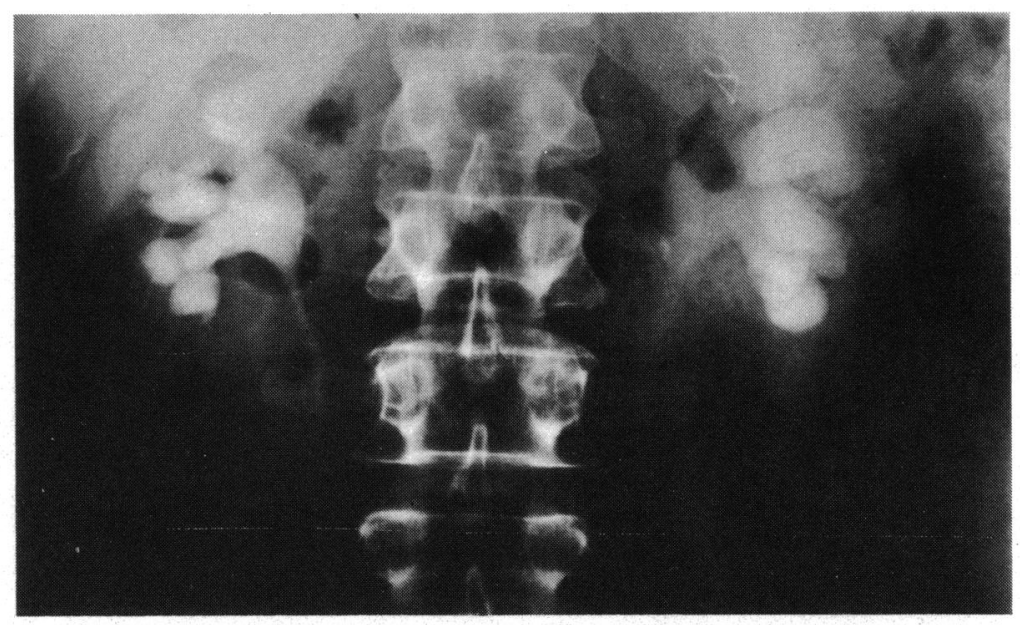

Fig. 3 Intravenous pyelogram illustrating moderate hydronephrosis on the right and severe hydronephrosis on the left from bilateral ureteropelvic obstructions.

after his discharge from the hospital. No proptosis or problems of extraocular motility were present during these examinations.

In July 1980 the patient was admitted to the hospital for investigation of a pelvic mass and haematuria. A pyelogram revealed obstruction of the right ureter at the pelvis, and the bladder appeared elevated and compressed. On 2 July 1980 cystoureteroscopy and a right pyeloureterogram were performed. Biopsy of a mass at 4 o'clock in the left sacrococcygeal wall was done through a proctoscope. The biopsy showed non-inflamed, benign, fibrous connective tissue (Fig. 2).

The patient was readmitted to hospital in May 1981 for investigation of bilateral progressive hydronephrosis and fever of unknown origin. During this spell in hospital he developed bilateral mild anterior uveitis, which responded to topical prednisone and mydriatic therapy. Right orbital congestion with $5 \mathrm{~mm}$ of proptosis and some limitation of extraocular motility also became apparent. Computerised tomography (CT) scans of the orbits confirmed the proptosis and demonstrated attenuation of the fat of the right orbit. There was slight enlargement of the extraocular muscles, but this was not thought to be characteristic of the myopathy accompanying thyroid disease.

On physical examination he was found to have an enlarged spleen and liver. However, CT scans of the abdomen and bipedal lymphangiograms were normal. An intravenous pyelogram now showed bilateral hydronephrosis (Fig. 3). Retrograde pyelograms showed an inflammatory process of the renal pelvis with obstuction at that level. The urine analysis was normal. The blood urea nitrogen (BUN) was 12 $\mathrm{mg} / \mathrm{dl}(2.0 \mathrm{mmol} / \mathrm{l})$ and serum creatinine was 1.4 $\mathrm{mg} / \mathrm{dl}(124 \mu \mathrm{mol} / \mathrm{l})$. Thyroid function tests showed a thyroxine radioimmunoassay of $6.8 \mu \mathrm{g} / \mathrm{dl}$ with thyroxine-binding globulin of $1.00 \mu \mathrm{g} / \mathrm{dl}$. (SI conversion: $\mu \mathrm{g}-\mathrm{dl} \times 10=\mu \mathrm{g} / \mathrm{l}$.) Thyroglobulin antibodies were normal at less $1: 10$ and microsomal $A B$ was normal at less than 1:100. The LATS-TSH was normal at $6 \mu \mathrm{l} / \mathrm{ml}(6 \mathrm{ml} / \mathrm{l})$.

On 26 May 1981 surgical lysis of retroperitoneal fibrosis with intaperitonisation of the ureters was carried out to relieve the bilateral hydronephrosis. The patient was found to have a 4-7 mm thick sheathlike fibrosis extending from just above the lower pole of each kidney to $2.5 \mathrm{~cm}$ below the iliac vessels. The fibrosis completely encased the ureters and extended over the great vessels but did not constrict them. The liver, spleen, and other abdominal organs were examined and found to be healthy and not involved in the fibrotic process. Biopsy from the retroperitoneal fibrotic-appearing tissue was found to be histologically consistent with the tissue changes observed in the 1980 biopsy (Fig. 4). He was treated with $50 \mathrm{mg}$ of prednisone daily following his surgical exploration. This resulted in resolution of the proptosis and ophthalmoplegia. The steroid therapy was tapered and stopped in July 1981. He was subsequently treated with a non-steroidal anti-inflammatory agent (ibuprofen $1600 \mathrm{mg}$ daily). This was discontinued in January 1983.

The patient remained asymptomatic until May 1983, when he again developed pain and swelling about his left eye. His left upper eyelid and superior orbit were red and tender, and there was limitation of upgaze. Mild anterior uveitis was also present in the left eye. The inflammation rapidly subsided on treatment with ibuprofen. His visual acuity remained $20 / 20$ and the visual fields were unaffected. An intravenous pyelogram was normal, and no systemic symptoms developed. When last seen in August 1983 
Fig. 4 Retroperitoneal biopsy showing fibrous tissue with some areas of chronic inflammatory cell infiltration. (Hand $E, \times 300)$.

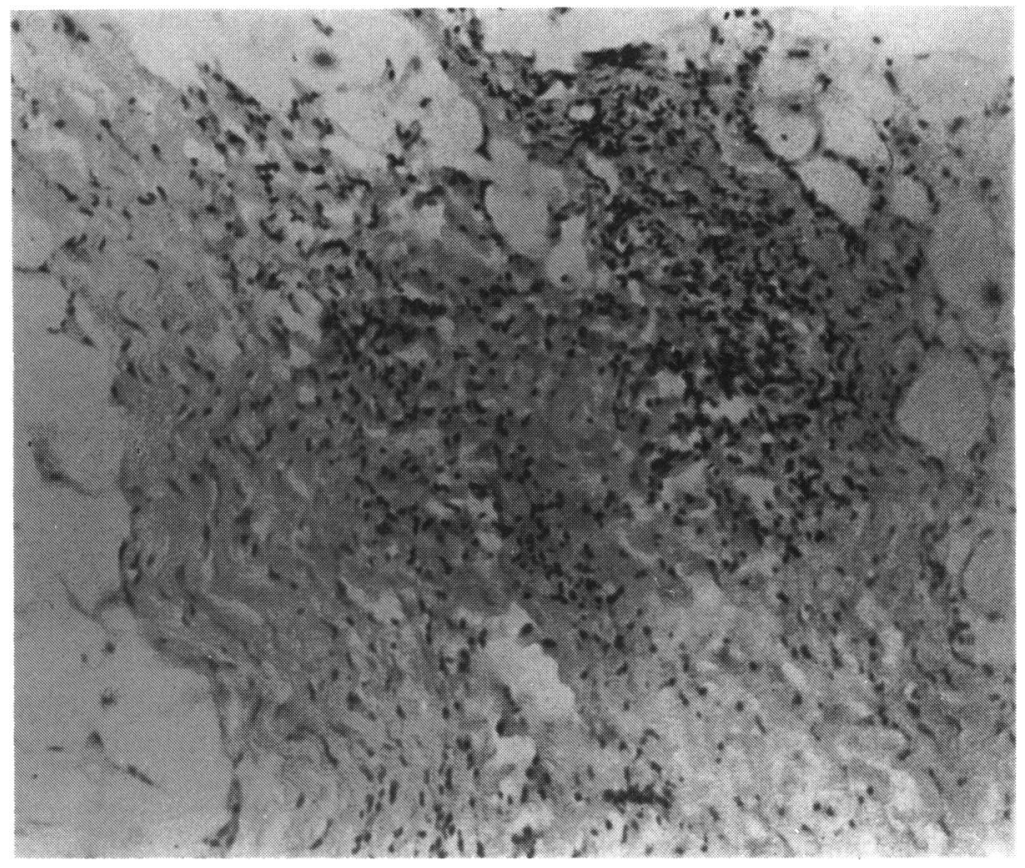

he was asymptomatic and was being treated with 1600 $\mathrm{mg}$ of ibuprofen daily.

\section{Discussion}

We are aware of three previously reported cases of orbital inflammation with proptosis (orbital pseudotumour) in association with retroperitoneal fibrosis and obstructive renal disease ${ }^{34}$ This entity was first described in 1967 by Comings and coworkers, who reported the ocular and abdominal findings in two brothers. ${ }^{3}$ Their first patient, a 37-year-old man, initially presented with symptoms of recurrent episcleritis, which 10 years later recurred in association with proptosis and with gastrointestinal symptoms of nausea, vomiting, and lower abdnominal pains. A non-functioning kidney and hydronephrosis were found. A sheet-like fibrosis covering the ureters was noted on exploratory laparotomy. Biospy showed dense fibrous tissue infiltrated with leucocytes and plasma cells. The patient's 39-year-old brother had recurrent episodes of arthralgia of the hands as a child. By the age of 18 he had progressive dyspnoea on exertion. The trachea and vena cava were found to be encased in a fibrous mass during exploratory thoracotomy. At the age of 33 exophthalmos of his right eye developed. An orbital biopsy was not obtained in either patient.

Richards and coworkers ${ }^{4}$ reported on a 66-year-old woman with a three-year history of declining health secondary to renal failure and a six-month history of painless proptosis of her right eye. Biopsy specimens of the orbit and of the abdomen were stated to be similar and to be composed of fibrous connective tissue interspersed with collections of acute and chronic inflammatory cells.

As we have noted in the introduction to this report, orbital inflammation and episcleritis associated with proptosis and limitation of ocular motility may occur in individuals with several forms of coexisting systemic inflammatory diseases. These may respond to treatment and resolve without residual fibrosis, but late scarring and limitation of eye movements often occur. Our patient has not shown clinical signs or laboratory findings consistent with any of these conditions. He has not developed postinflammatory orbital fibrosis, and the orbital biopsy showed no evidence of necrosis or of scarring. Nevertheless we assume that a relationship exists between his recurrent ocular inflammatory episodes and his retroperitoneal fibrotic lesions.

The pathogenesis of multifocal fibrosclerosis is unknown. It has been proposed that the retroperitoneal fibrotic component represents a localised secondary response to gastrointestinal inflammation." A similar retroperitoneal process may be initiated by the ingestion of methysergide. ${ }^{\circ}$ Retroperitoneal fibrosis causing extrinsic ureteral obstruction was first described by Albarran.' The fibrous process may exist without creating ureteral obstruc- 
tion, but the ureter is quite vulnerable to compression and distortion by the fibrosis and is therefore the structure most frequently affected. The inferior vena cava and aorta may also be involved. ${ }^{*}$

In our case the retroperitoneal mass was described by the surgeon as a fibrotic wall, and biopsy specimens showed only thickened fibrotic tissue. Specimens with these characteristics may initially be regarded as being inadequate, especially in cases where a malignancy is suspected because of the obstructive nature of the disease. Care must be taken to make certain that adequate specimens are obtained from different sites and that the process is indeed benign, since a similar fibrotic process may be seen with carcinoma of the sigmoid, carcinoid tumours, reticulum cell sarcoma, and Hodgkin's disease. ${ }^{5}$ Once the benign nature of the obstructive disease is established, intraperitoneal transplantations of the ureters to relieve the urinary obstruction has been recommended. This procedure was successful in our patient, and he is now asymptomatic. The patient's mother had a past history of surgery for oesophageal obstruction; this suggests a possible familial incidence. However, the histological diagnosis of her chest lesion is unknown; there has been no recurrence of her disease, and she has not shown ocular or other abnormalities of the type associated with multifocal fibrosclerosis.
It appears that the use of non-steroidal antiinflammatory agents has been beneficial in suppressing recurrence of this patient's orbital inflammation. He has had only one recurrence since his hospitalisation, but this occurred while he was off his medication. Because of the short follow-up interval in this case the long-term benefit of this form of therapy is uncertain.

\section{References}

1 Duke-Elder S. System of ophthalmology. St Louis: Mosby, 1974; 13(2): 892.

2 Jakobiec FA, Jones IS. Orbital inflammation. In: Duane TD, ed. Clinical ophthalmology. Hagerstown: Harper and Row, 1975: 2: chapter 25 .

3 Comings DE, Skubi KB, Van Eyes J, Motulsky AG. Familial multifocal fibrosclerosis. Ann Intern Med 1962; 66: 884-92.

4 Richards AB, Skalka HW, Roberts FJ, Flint A. Pseudotumor of the orbit and retroperitoneal fibrosis. Arch Ophthalmol 1980; 98: 1617-20.

5 Hache L, Utz DC, Woolmer LB. Idiopathic fibrosis retroperitonitis. Surg Gynecol Obstet 1962; 115: 737-44.

6 Graham JR. Methylsergide for prevention of headache: experience in 500 patients over three years. $N$ Engl $J$ Med 1964; 270: 67-72.

7 Albarran J. Retention renale por perc ureterite: liberation externa de l'uretere. Assoc Fr Urol 9: 511-520 (1905).

8 Niclsen HK. Multifocal idiopathic fibrosclerosis. Acta Med Scand 1980; 208: 119-23. 